\title{
Disorganisation: a model for 'early amnion rupture'?
}

\author{
D DONNAI* AND R M WINTER† \\ From *the Regional Genetic Centre, St Mary's Hospital, Manchester M13 0JH; and the Kennedy Galton \\ Centre, Northwick Park Hospital, Harrow, Middlesex HAl $3 U J$.
}

SUMmaRY The hypothesis of an intrinsic defect of germ plasma put forward by Streeter to explain the abnormalities observed in 'amniotic bands sequence' (ABS) was not supported by Torpin, who suggested that the bands derived from early amnion rupture, with formation of mesodermal strings and naked chorion, which then constricted or adhered to fetal parts. Recently, several authors have recorded and discussed possible mechanisms for 'non-band related' malformations in patients with otherwise typical limb constrictions and amputations.

The mouse mutant disorganisation (Ds) is a semidominant with $72 \%$ of heterozygotes manifesting abnormalities, which include cranioschisis, limb duplications and deficiencies, gastro/thoracoschisis, and papillae protruding from other parts of the body. We report similar abnormalities including papillae in five fetuses and one newborn with ABS and, based on these observations and published reports, we suggest that a human homologue for Ds may be the cause of at least some examples of ABS.

Streeter ${ }^{1}$ examined embryos and concluded that ring constrictions are localised areas of imperfectly formed tissue owing to defective areas of germ plasma which, because of the proximity to the developing amnion, caused involvement of this structure too. Later, Torpin ${ }^{2}$ suggested that premature rupture of the amnion was responsible for congenital ring constrictions and amputations. The mechanism suggested was that, after rupture of the amnion and separation from the chorion, mesodermal strings formed which constricted fetal parts, the naked chorion acting as a site to which fetal parts could adhere, this process being helped by amniotic fluid leakage. This latter hypothesis has been generally accepted and observations of anomalies not easily explained by amniotic band disruption have been attributed to either vascular interference or mechanical teratogenesis. We report five fetuses and one newborn, all with anomalies commonly seen in ABS, and in addition anomalies difficult to explain by amniotic band disruption. The mouse mutant disorganisation (Ds) has anomalies which are very similar to those observed in ABS fetuses and we suggest that a human homologue of Ds may be responsible for some cases of ABS and may explain the rare familial reports of apparent ABS.

Received for publication 7 February 1989.

Acrepted for publication 9 February 1989.

\section{Case reports}

CASE 1

This was a female fetus aborted at 21 weeks' gestation by a 29 year old primigravid mother who had raised serum $\alpha$ fetoprotein (AFP) and amniotic fluid AFP. The fetus had a large encephalocele in the frontoparietal area and clefting of the left side of the face with an exophthalmic right eye. There were multiple amputations of the digits of the hands and feet with terminal syndactyly. The umbilical cord, at the insertion into the abdomen, contained three vessels, but $3.5 \mathrm{~cm}$ distally it divided, sending two vessels to the placenta and one vessel to the meninges covering the encephalocele. There were three skin tags containing cartilage $0.5 \mathrm{~cm}$ long over the left shoulder and upper cervical region (fig 1).

\section{CASE 2}

This was a female fetus aborted by a 28 year old primigravid woman after the detection of high serum and amniotic fluid AFP levels. The fetus had a huge right sided thoraco/gastroschisis; membrane bands extended from the cord and fetal surface of the placenta to the back of the abdominal defect and to the liver. There were amputations of the fingers with distal syndactyly. There was a right primary cleft lip and palate and a short, thick neck, but no tissue bands observed in the facial region. There was a blister over the back. A skin spur projected from 
the left calf and there was angulation of the right tibia and fibula. The right hallux was duplicated with a deep cleft between it and the second toe. The left kidney was absent and there was a single umbilical artery (fig 2).

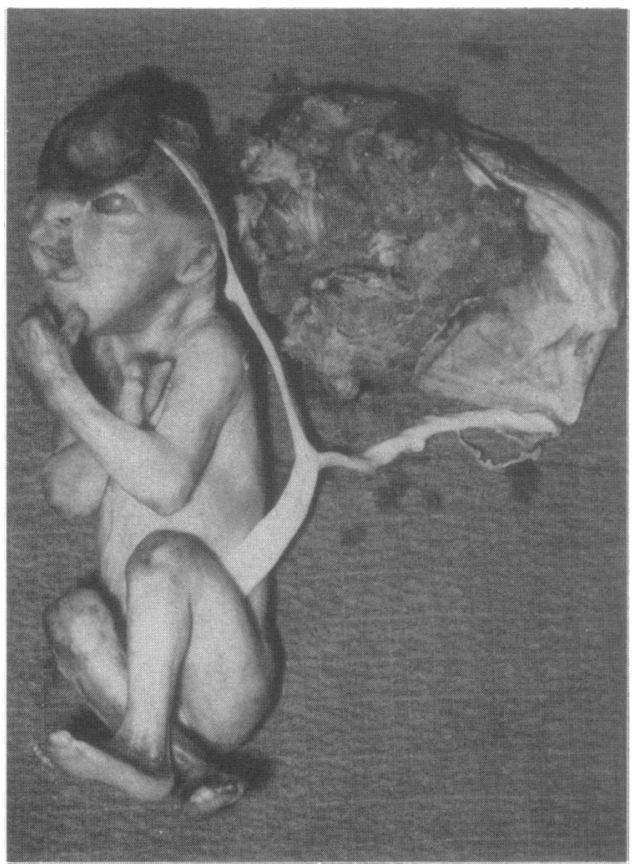

FIG 1 Case 1; note distal amputations, divided umbilical cord, and skin tag on left shoulder.
CASE 3

This was a female fetus aborted at 18 weeks' gestation in the first pregnancy of a 20 year old woman. There was a large defect involving the right parietal and occipital bone, a primary bilateral cleft lip and palate, and a skin spur over the mid thoracic region posteriorly. There were amputation deformities with terminal syndactyly of the fingers. There was complete syndactyly of the fourth and fifth toes of both feet.

\section{CASE 4}

This was a male fetus aborted at 18 weeks' gestation by a 22 year old normal woman, whose older son has unilateral renal agenesis. Ultrasound scan in the pregnancy had shown a lack of liquor and severe fetal distortion. The placenta was attached to an abdominal wall defect in the fetus and no umbilical cord was identified. The trunk was rotated through $100^{\circ}$ and the legs compressed against the back. The right arm was hypoplastic with very short upper and lower segments, with webbing across the antecubital fossa. The hand was represented by a nub of tissue. There was hydrocephalus and an occipital skull defect. There appeared to be three nostrils present, two on the right separated by a groove from one on the left (fig 3).

\section{CASE 5}

This was a female fetus aborted by a 18 year old primigravida after ultrasound scan diagnosis of hydrocephalus. There were amputations of the fingers of both hands with terminal syndactyly with tissue strands. Both frontal bones were deficient as

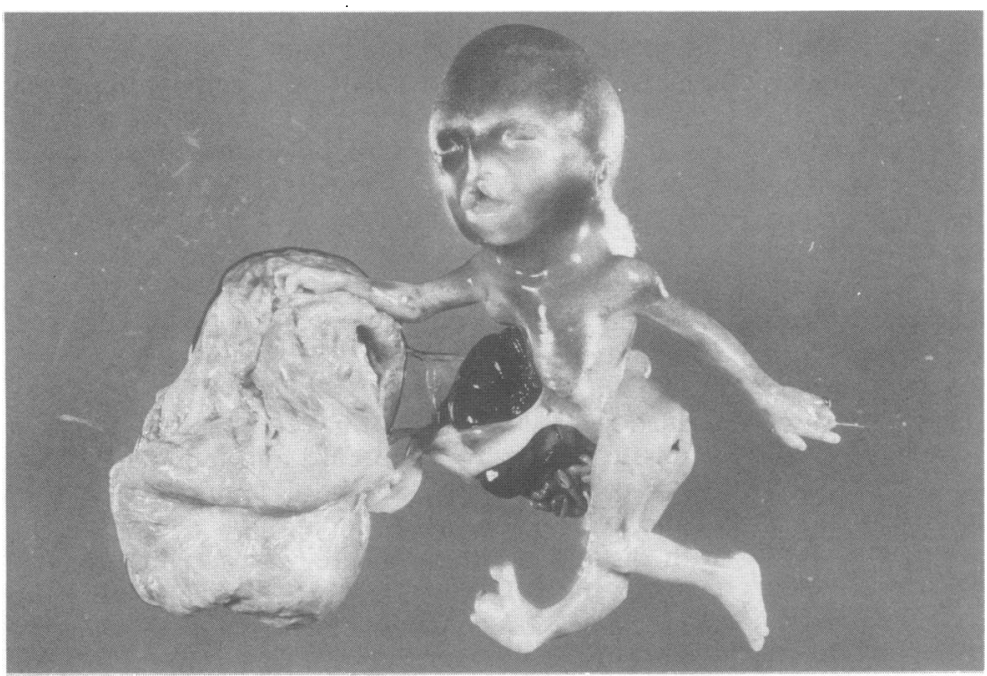

FIG 2 Case 2; note primary cleft lip, amputation of fingers on left hand, blister on back, skin spur on left calf, and preaxial polydactyly of right foot. 
was the ethmoid plate. The anterior falx was absent and there was wide separation of the nostrils with a groove extending upwards to a skin spur projecting from the region of the anterior fontanelle (fig 4). There was bilateral microphthalmos.

CASE 6

This girl is the offspring of unrelated Caucasian parents. The family and pregnancy history were unremarkable. At birth she was noted to have a papilla over the left temporal region that appeared

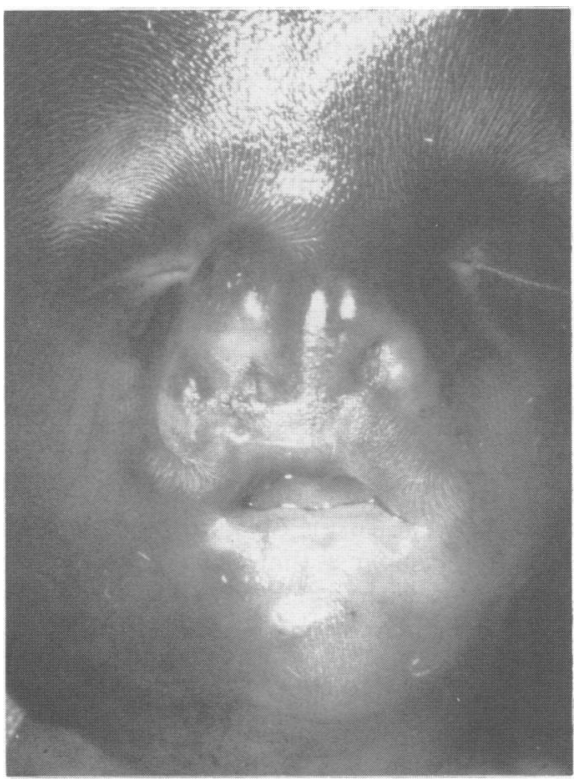

FIG 3 Case 4; note presence of three nostrils.

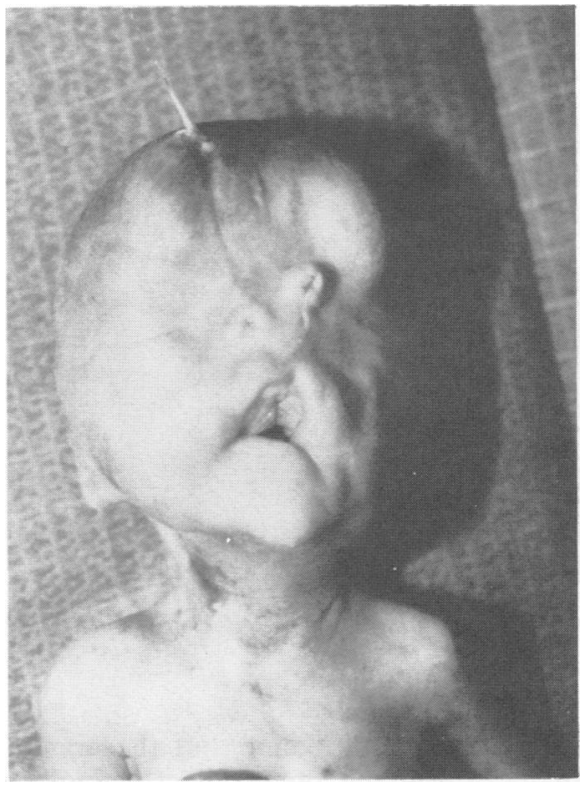

FIG 4 Case 5; note separated nostrils, widely spaced eyes, and skin spur over anterior fontanelle.

to be tethered to the underlying muscle, because it was retractile when she cried (fig 5). There was a similarly tethered sinus over the right temporal region. A small occipital encephalocele was noted and a CT scan of the brain showed hydrocephalus. There were absent fingers with a remaining thumb and index finger on the right hand (fig 6) and a ring constriction of the thumb of the left hand. A renal ultrasound scan showed a possible unilateral cystic kidney. The hand abnormalities appeared to be

FIG 5 Case 6 with papilla on the left temple. 


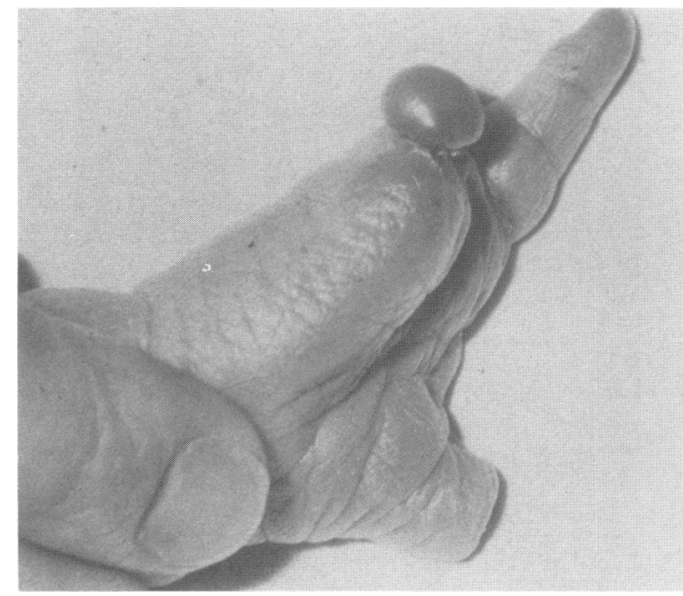

FIG 6 Case 6; amputation of digits 3 to 5 on right hand.

secondary to amniotic bands, but the craniofacial abnormalities and the renal defect cannot be explained on this basis.

\section{Discussion}

There are many published reports of fetuses and infants with ABS who in addition have anomalies difficult to explain by band disruption. Higginbottom $e t a^{3}$ described a study where two of the cases had primary cleft lip and palate, one an absent arm, shoulder girdle, and subclavian artery, and another choanal atresia and microphthalmos. It was suggested that these findings supported very early amnion rupture, that is, before fusion of the maxillary and median nasal processes and before limb budding. Graham et al $l^{4}$ described two babies with preaxial polydactyly as well as other band related defects. They suggested that the supernumerary digits resulted from compression of the developing foot plate before 42 days of development. Pagon et al, ${ }^{5}$ reporting 15 patients with limb/body wall defects, described abnormalities difficult to explain by band disruption, including absent limbs and kidneys, imperforate anus, micro/ anophthalmos, primary cleft lip and palate, lobster claw, and skin tags containing cartilage.

Recently, Hunter and Carpenter ${ }^{\circ}$ described four cases of $\mathrm{ABS}$ with malformations not resulting from amniotic bands and discussed previously suggested mechanisms; they concluded that the most likely hypothesis was an extrinsic insult or perhaps occasionally a familial susceptibility leading to loss of fetal vascular integrity. This in turn leads to superficial haemorrhage and denudation with adhesions causing syndactyly and constrictions, and also to the observed internal anomalies.

The mouse mutant disorganisation (Ds) has been described by Hummel. ${ }^{7}$ It arose as a spontaneous mutation in an inbred strain of mice and has been studied in heterozygotes. It appears to be a semidominant, lethal in homozygotes, and with incomplete penetrance in heterozygotes, $72 \%$ of which manifest anomalies. The effects of the mutation, while they affect development of structures derived from all germ layers and appear to be active during the whole period of embryogenesis, do seem to operate after the establishment of the primary axis. In 500 abnormal mouse fetuses, known by parental genotype to be heterozygotes, $67 \%$ had multiple defects and the rest single defects: $53 \%$ had cranioschisis or exencephaly; $40 \%$ had hamartomas represented by papillae of variable size and shape protruding from the body, some containing cartilage; and $33 \%$ had limb abnormalities, usually single and consisting of duplications or reductions, and sometimes separate but imperfect accessory limbs or digits. About $20 \%$ of affected fetuses had gastro- or thoracoschisis and $20 \%$ a facial abnormality, such as separation of the nasal processes. A smaller proportion had anal atresia, bladder exstrophy, and absent kidneys.

Our patients have anomalies which have been assumed to be the result of disruption by amniotic bands, and we suggest that these cases and those published cases with apparent ABS, in addition to anomalies difficult to explain by band disruption, may result from a mutant gene, the homologue of the mouse mutant disorganisation.

There are a few published reports of familial amniotic bands. Etches et al ${ }^{8}$ described a mother, child, and great aunt who all had amputations similar to those seen in ABS, and Lubinsky et al described two further families.

These familial instances could be explicable by the effects of a semidominant gene, such as Ds. While not suggesting that all cases of ABS are caused by a mutant gene homologous to Ds, the possibility should be borne in mind and an appropriate family history taken, specifically noting craniofacial, limb, and genitourinary anomalies.

\footnotetext{
References

1 Streeter GL. Focal deficiencies in fetal tissues and their relation to intrauterine amputation. Contr Embryol Carneg Instn 1930; 22: $1-44$.

2 Torpin R. Amniochorionic mesoblastic fibrous strings and amniotic bands. Associated constricting fetal malformations or fetal death. Am J Obstet Gynecol 1965;91:65-75.

3 Higginbottom MC, Jones KL, Hall BD, Smith DW. The amniotic band disruption complex: timing of amniotic rupture
} 
and variable spectra of consequent defects. $J$ Pediatr 1979;95: 544-9.

4 Graham JM, Higginbottom MC, Smith DW. Preaxial polydactyly of the foot associated with early amnion rupture: evidence for mechanical teratogenesis? J Pediatr 1981;98:943-5.

5 Pagon RA, Stephans TD, McGillivray BC, et al. Body wall defects with reduction limb anomalies: a report of fifteen cases. Birth Defects 1979;15(5A):171-85.

6 Hunter AGW, Carpenter BF. Implications of malformations not due to amniotic bands in the amniotic band sequence. Am J Med Genet 1986;24:691-700.
${ }^{7}$ Hummel KP. Developmental anomalies in mice resulting from action of the gene Disorganization, a semi-dominant lethal. Pediatrics 1959;23:212-21.

8 Etches PL, Stewart AR, Ives EJ. Familial congenital amputations. J Pediatr 1982;101:448-9.

9 Lubinsky M, Sujansky E, Sanger W, Salyards P, Severn C. Familial amniotic bands. Am J Med Genet 1983;14:81-7.

Correspondence to Dr D Donnai, Regional Genetic Centre, St Mary's Hospital, Manchester M13 0JH. 